\title{
Visualization System of the KAERI-DySCo for Dynamic Simulation of the VHTR-SI Process
}

\author{
Jiwoon Chang \\ Korea Atomic Energy \\ Research Institute, \\ jwjang73@kaeri.re.kr
}

\author{
Youngjoon Shin \\ Korea Atomic Energy \\ Research Institute, \\ nyjshin@kaeri.re.kr
}

\author{
Youn Cheong \\ Chungnam National \\ University \\ cyoun@cs.cnu.ac.kr
}

\author{
Taehoon Lee \\ Korea Atomic Energy \\ Research Institute, \\ leeth@kaeri.re.kr
}

\author{
Kiyoung Lee \\ Korea Atomic Energy \\ Research Institute, \\ kylee@kaeri.re.kr
}

\section{Yongwan Kim \\ Korea Atomic Energy \\ Research Institute, \\ ywkim@kaeri.re.kr}

Jonghwa Chang

Korea Atomic Energy

Research Institute

jhchang@kaeri.re.kr

\section{INTRODUCTION}

The Korea Atomic Energy Research Institute Dynamic Simulation Code (KAERI-DySCo) based on the Visual C++ is integration application software that simulates the dynamic behavior of the SI process (Shin, 2007). KAERI DySCo was prepared to solve dynamic problems of the seven chemical reactors in the VHTR-SI process (Shin, et al., 2009). By using this computer code, much complex and variety raw data such as thermodynamics data, mole flow rates of input and output materials and their temperature and pressure information can be generated. In order to effectively classify and monitor a lot of raw data generated from the dynamic simulation of the SI process, a proper visualization system is required in KAERI-DySCo.

In this paper, software systems using Chart FX and Spread 7.0 to visualize the raw data generated from the dynamic simulation of the SI process are introduced. The Chart FX has been used to represent the raw data in the form of a graphical chart. To apply the Chart FX which is based on the .NET platform into KAERI-DySCo which is based on the Microsoft Foundation Class (MFC) platform of the Visual C++, a Windows Forms Control Hosting method has been adopted. On the other hand, the Spread 7.0 has been used to present the raw data with a numerical table type. The Spread 7.0 has been embedded in KAERI-DySCo by using the Active-X method.

\section{EXAMPLE OF DATA VISUALIZATION}

As a representative example of the applied data visualization in KAERI-DySCo, the main window and the sub window are shown in Fig.1 and 2. The main window that includes the thermal pathway of the VHTR-SI process has 23 spreadsheets to correspond to the process line number. The sub window has seven dynamic response charts. The charts display the mole flow rate and temperature values as a function of time.

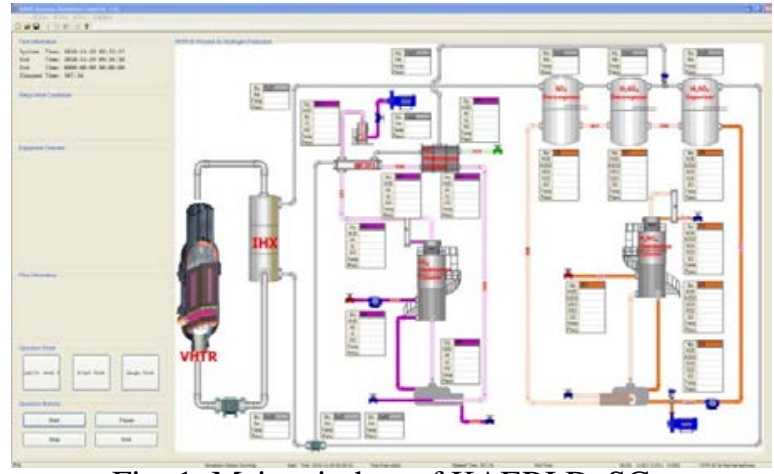

Fig. 1. Main window of KAERI DySCo.

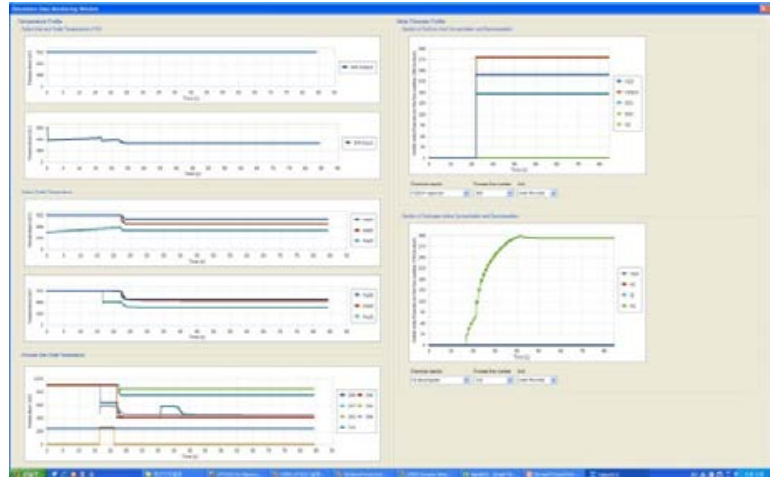

Fig. 2. Sub window of KAERI DySCo.

\section{CONCLUSIONS}

The visual dynamic information displayed by a main window and sub windows of KAERI-DySCo allow users to easily analyze the start-up dynamic behavior of the SI process coupled to the VHTR.

\section{REFERENCES}

Shin, Youngjoon, 2007, Calculation Note of the NHDD (NHDD-KA07-HP-001-00).

Shin, Youngjoon, et al., 2009, Nuclear Engineering and Technology, Vol. 41, pp 831-840.

Copyright (C) 2011 by JSME 


\section{Visualization System of the KAERI-DySCo for Dynamic Simulation of the VHTR-SI Process}

\author{
Jiwoon Chang \\ Korea Atomic Energy \\ Research Institute, \\ 1045 Daedeok-daero, \\ Yuseong-gu, Daejeon \\ 305-353, Republic of Korea \\ Phone: +82-42-868-8369 \\ jwjang73@kaeri.re.kr
}

\author{
Youngjoon Shin \\ Korea Atomic Energy \\ Research Institute, \\ 1045 Daedeok-daero, \\ Yuseong-gu, Daejeon \\ 305-353, Republic of Korea \\ Phone: +82-42-868-2795 \\ nyjshin@kaeri.re.kr
}

Kiyoung Lee
Korea Atomic Energy
Research Institute,
1045 Daedeok-daero,
Yuseong-gu, Daejeon
305-353, Republic of Korea
Phone: +82-42-868-2807
kylee@kaeri.re.kr

Jonghwa Chang
Korea Atomic Energy
Research Institute
1045 Daedeok-daero,
Yuseong-gu, Daejeon
305-353, Republic of Korea
Phone: +82-42-868-2884
jhchang@kaeri.re.kr

\author{
Youn Cheong \\ Chungnam National \\ University \\ 220 Gung-dong, \\ Yuseong-gu, Daejeon \\ 305-764, Republic of Korea \\ Phone: +82-42-821-6275 \\ cyoun@cs.cnu.ac.kr
}

\author{
Yongwan Kim \\ Korea Atomic Energy \\ Research Institute, \\ 1045 Daedeok-daero, \\ Yuseong-gu, Daejeon \\ 305-353, Republic of Korea \\ Phone: +82-42-868-8981 \\ ywkim@kaeri.re.kr
}

Keywords: VHTR-SI process, Hydrogen production, Dynamic simulation, Visualization, Chart, Spreadsheet

\begin{abstract}
The Korea Atomic Energy Research Institute-Dynamic Simulation Code (KAERI-DySCo) based on the Visual C++ which is an integration application software to analyze the dynamic behavior of the Sulfur Iodine (SI) process, which is a nuclear hydrogen process that is coupled to a Very High Temperature Gas Cooled Reactor (VHTR) through an Intermediate Heat Exchanger (IHX) generates a large number of raw data during its execution process. The generated raw data include various types of data such as thermodynamics data, mole flow rates of input and output material and their temperature and pressure information. In order to effectively classify and monitor the generated raw data, data
\end{abstract}

visualization is required. As tools of the data visualization, the Chart FX and the Spread 7.0, which are commercial components, have been used; this has been used to process database of the raw data in the form of numerical value, and that has been used to present the raw data in the form of a graphical chart. The Chart FX based on the .NET platform is embedded in the KAERI-DySCo in form of a Dynamic Link Library (DLL) by using a Windows Forms Control Hosting method. On the other hand, the Spread 7.0 is embedded in the KAERI-DySCo by using an ActiveX control method. As results of the data visualization, a main window of the KAERI-DySCo and its sub window have been introduced. A start-up dynamic simulation result of a HIx distillation 
column by using the KAERI-DySCo has been also introduced as an example of data visualization output.

\section{INTRODUCTION}

The SI process coupled to the VHTR is well known as a feasible technology to produce hydrogen. The SI process can be divided into three sections based on the chemical reactions: Bunsen reaction (Section 1), sulfuric acid concentration and decomposition (Section 2), and hydriodic acid concentration and decomposition (Section 3) as the following chemical reactions.

$$
\begin{aligned}
& \mathrm{I}_{2}+\mathrm{SO}_{2}+2 \mathrm{H}_{2} \mathrm{O} \rightarrow 2 \mathrm{HI}+\mathrm{H}_{2} \mathrm{SO}_{4}\left(\sim 120{ }^{\circ} \mathrm{C}\right) . \\
& \mathrm{H}_{2} \mathrm{SO}_{4} \rightarrow \mathrm{H}_{2} \mathrm{O}+\mathrm{SO}_{2}+1 / 2 \mathrm{O}_{2}\left(\sim 850{ }^{\circ} \mathrm{C}\right) . \\
& 2 \mathrm{HI} \rightarrow \mathrm{H}_{2}+\mathrm{I}_{2}\left(400 \sim 500{ }^{\circ} \mathrm{C}\right) .
\end{aligned}
$$

The heat required in the SI process can be supplied through an intermediate heat exchanger (IHX) by the VHTR. Helium is used as a high temperature energy carrier gas between the VHTR and the IHX or the IHX and the SI process. In the SI process, chemical reactors that receive thermal energy directly from the helium are: A sulfuric acid vaporizer, a sulfuric acid decomposer, a sulfur trioxide decomposer, and a hydriodic acid decomposer including pre-heating part. On the other hand, two reboilers for the $\mathrm{H}_{2} \mathrm{SO}_{4}$ and $\mathrm{HI}$ distillation columns are heated by the sensible or latent heat of the SI process stream (Shin, 2007).

The Korea Atomic Energy Research Institute-Dynamic Simulation Code (KAERI-DySCo) based on the Visual C++ is integration application software that simulates the dynamic behavior of the SI process. KAERI DySCo was prepared to solve dynamic problems of the seven chemical reactors mentioned above (Kim, et al., 2008) (Shin, et al., 2009). By using this computer code, much complex and variety raw data such as thermodynamics data, mole flow rates of input and output materials and their temperature and pressure information can be generated. In order to effectively classify and monitor a lot of raw data generated from the dynamic simulation of the SI process, a proper visualization system is required in KAERI DySCo.

In this paper, software systems using Chart FX and Spread 7.0 to visualize the raw data generated from the dynamic simulation of the SI process are introduced. The Chart FX has been used to represent the raw data in the form of a graphical chart. To apply the Chart FX which is based on the .NET platform into KAERI-DySCo which is based on the Microsoft Foundation Class (MFC) platform of the Visual $\mathrm{C}++$, a Windows Forms Control Hosting method has been adopted. On the other hand, the Spread 7.0 has been used to present the raw data with a numerical table type. The Spread 7.0 has been embedded in KAERI DySCo by using the Active-X method.

\section{THERMAL PATHWAY}

The key chemical reactors in the section 1 are a Bunsen reactor which has not only a function of a Bunsen reaction, but also a function of a mutual separation of the immiscible sulfuric acid and hydrogen iodide phases generated from the Bunsen reaction, a sulfuric acid stripper to remove the residual sulfuric acid from the separated hydrogen iodide phase, and a sulfur dioxide scrubber to recover the sulfur dioxide from the oxygen/sulfur dioxide mixture. These reactors do not require thermal energy from an external thermal source.

Section 2 consists of a sulfuric acid distillation column, a sulfuric acid vaporizer, a sulfuric acid decomposer, and a sulfur trioxide decomposer. All of the chemical reactors need a heat supply from an external heat source. When an array of these reactors is determined by the operational temperature order, the high temperature helium to heat these reactors has to go through the sulfur trioxide and sulfuric acid decomposers and the vaporizer in series. The cooled helium vented from the vaporizer is recycled to an intermediate heat exchanger (IHX) to receive thermal energy. On the other hand, the sulfuric acid distillation column that operates at a lower temperature can be heated by the sensible heat of a process gas as shown in Fig. 1

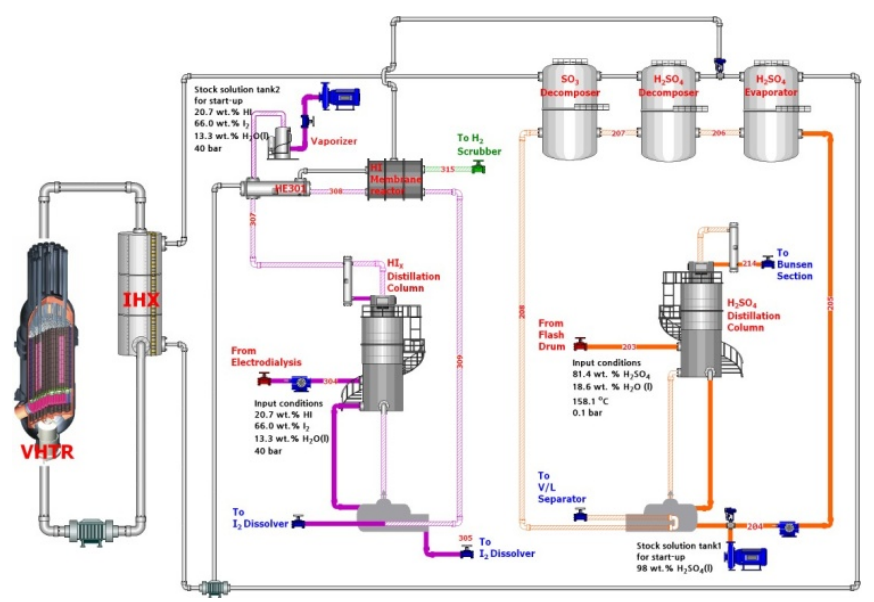

Fig. 1. Thermal pathway of the SI process coupled to a VHTR.

Section 3 has an electrodialysis equipment to preliminarily concentrate the HIx solution, an HIx solution distillation column for an additional concentration and a vaporization of the HIx solution. An HI decomposer has two functions of a catalysis decomposition of $\mathrm{HI}$ and $\mathrm{a}$ preferential separation of hydrogen from the decomposed gas mixture of $\mathrm{H}_{2} / \mathrm{I}_{2} / \mathrm{HI} / \mathrm{H}_{2} \mathrm{O}$. The $\mathrm{HI}$ decomposer is heated by circulated helium and the HIx distillation column is heated by the sensible heat of another process gas.

\section{Structure of KAERI-DySCo}

In order to analyze the dynamic behavior of the VHTR-SI process shown in Fig. 1, KAERI-DySCo has been prepared to solve mathematical model equations by using numerical solvers and thermodynamics data. The program also supports a user-friendly graphical user interface (GUI) for user input and out. This functional architecture of KAERI-DySCo is shown Fig. 2 (Chang, et al., 2008).

Object-oriented analysis (OOA) and Object-orient design (OOD) methodologies were employed in this computer code and the program has a MFC framework structure based on the Visual $\mathrm{C}++$. There are specific user-defined classes that correspond to seven chemical reactors on the VHTR-SI thermal pathway. The user-defined classes are shown in Copyright (C) 2011 by JSME 
Table 1.

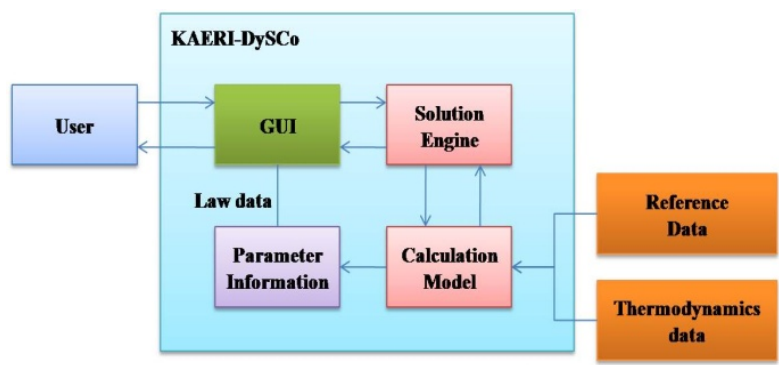

Fig. 2. Functional architecture of KAERI-DySCo.

Table 1. Lists of the key user-define classes

\begin{tabular}{|l|l|}
\hline \multicolumn{1}{|c|}{ Class name } & \multicolumn{1}{c|}{ Object reactor } \\
\hline CSADistillation & $\mathrm{H}_{2} \mathrm{SO}_{4}$ distillation column \\
\hline CH2SO4Evaporator & $\mathrm{H}_{2} \mathrm{SO}_{4}$ vaporizer \\
\hline CH2SO4Decomposer & $\mathrm{H}_{2} \mathrm{SO}_{4}$ decomposer \\
\hline CSO3Decomposer & $\mathrm{SO}_{3}$ decomposer \\
\hline CHIxDistillation & $\mathrm{HIx}$ distillation column \\
\hline CHIHeatExchanger & $\mathrm{HI}$ pre-heater \\
\hline CHIDecomposition & $\mathrm{HI}$ decomposer \\
\hline
\end{tabular}

The listed classes in Table 1 are referred to each other by a call-by-reference method during the program execution. The call-by-reference method has the advantage of greater timeand space-efficiency, as well as the potential for greater communication a class and its caller. On the other hand, key member variables in the each user-defined class, which can be converted into raw data, widely consist of mole flow rates of input and output materials and their temperatures and pressures. In addition, the important thermodynamics data, which are reused or referred among the each class, were also declared as the member variable. In naming rule of the member variables, common names have been used to improve code visibility and to easily classify input and output data. Table 2 shows the common names and their descriptions in naming rule of the member variables.

Table 2. Lists of common name in the member variables

\begin{tabular}{|c|c|}
\hline Common name & Description \\
\hline $\begin{array}{l}\text { pg_inputs_mole_f_ } \\
\text { pg_output_mole_f_ }\end{array}$ & $\begin{array}{l}\text { Input and output mole flow rate } \\
\text { of process gas }\end{array}$ \\
\hline $\begin{array}{l}\text { pg_inputs_temp_c_- } \\
\text { pg_output_temp_c_- }\end{array}$ & $\begin{array}{l}\text { Input and output temperature of } \\
\text { process gas }\end{array}$ \\
\hline $\begin{array}{l}\text { he_inputs_mole_f_- } \\
\text { he_output_mole_f_- }\end{array}$ & $\begin{array}{l}\text { Input and output mole flow rate } \\
\text { of helium gas }\end{array}$ \\
\hline $\begin{array}{l}\text { he_inputs_temp_c_ } \\
\text { he_output_temp_c__ }\end{array}$ & $\begin{array}{l}\text { Input and output temperature of } \\
\text { helium gas }\end{array}$ \\
\hline
\end{tabular}

\section{Methodologies of the Data Visualization}

The object of this study is to visualize the raw data, which can be generated by the KAERI-DySCo execution. The raw data, as stated earlier, include various types of data such as thermodynamics data, mole flow rates of input and output material and their temperature and pressure information. As a way to visualization of these data, we have used two commercial components that can be embedded in the KAERI-DySCo. One is the Chart FX of SoftwareFX, Inc. to accomplish a graphical charting of the data, and the other is the Spread 7.0 of FarPoint Technologies, Inc. to process the database of the data.

\subsection{Window Forms Control Hosting Method}

In the case of the Chart FX, it is based on the Microsoft's Visual Studio .NET. Therefore, a specific method such as the window forms control hosting method is needed to apply the KAERI-DySCo based on the Microsoft's Visual Studio C++. The windows forms control hosting method is divided into two procedures: a creating procedure of the .NET user control (such as the Chart FX) and a set-up procedure of the Visual C++ (Microsoft Corporation, 2008).

A flow chart for the window forms control hosting method is shown in Fig. 3. In Fig. 3, the left side represents a creating procedure of the .NET user control, and the right side represents a setup procedure of the Visual $\mathrm{C}++$.

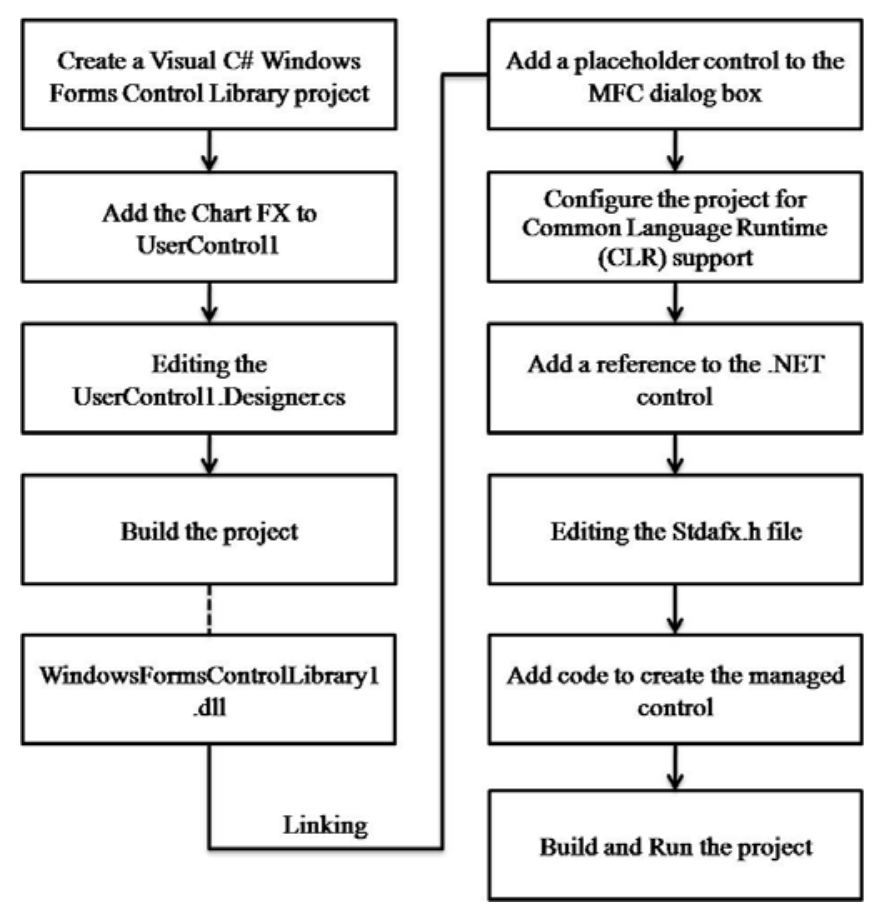

Fig. 3. Flow chart for the windows forms control hosting method.

\subsection{MFC ActiveX Controls}

An ActiveX control is a reusable software component based on the Component Object Model (COM) that supports a wide variety of OLE functionality and can be customized to fit many software needs. An ActiveX control can draw itself in its own window, respond to events (such as mouse clicks), and be managed through an interface that includes properties and methods similar to those in Automation Objects. These controls can be developed for many uses such as database access, data monitoring, or graphing.

Unlike the Chart FX, the Spread 7.0, which supports MFC platform, can be directly inserted into the KAERI-DySCo without a specific procedure.

\subsection{Example of the Data Visualization}

There are two analysis modes in KAERI-DySCo. One is the numeric mode by using the Spread 7.0, and the other is 
the graph mode by using the Chart FX. In case of the numeric mode, the spreadsheets, which have two columns and a maximum of eight rows, have been positioned on the thermal pathway main window showing the SI process coupled to the VHTR to monitor the dynamic vales of each chemical reactor as a function of time such as mole flow rate, temperature, and pressure. On the other hand, in the graph mode, the dynamic response chart of each reactor can be displayed on the sub window of KAERI-DySCo to analyze its dynamic pattern in detail.

Figs. 4 and 5 show the main window and the sub window. The main window that includes the thermal pathway of the VHTR-SI process has 23 spreadsheets to correspond to the process line number. The sub window shown in Fig. 5 has seven dynamic response charts. The charts display the mole flow rate and temperature values as a function of time.

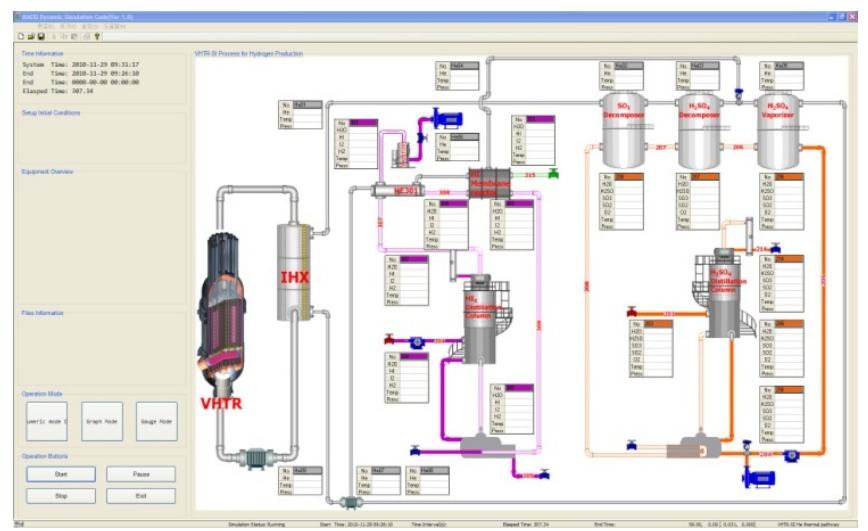

Fig. 4. Main window of KAERI-DySCo.

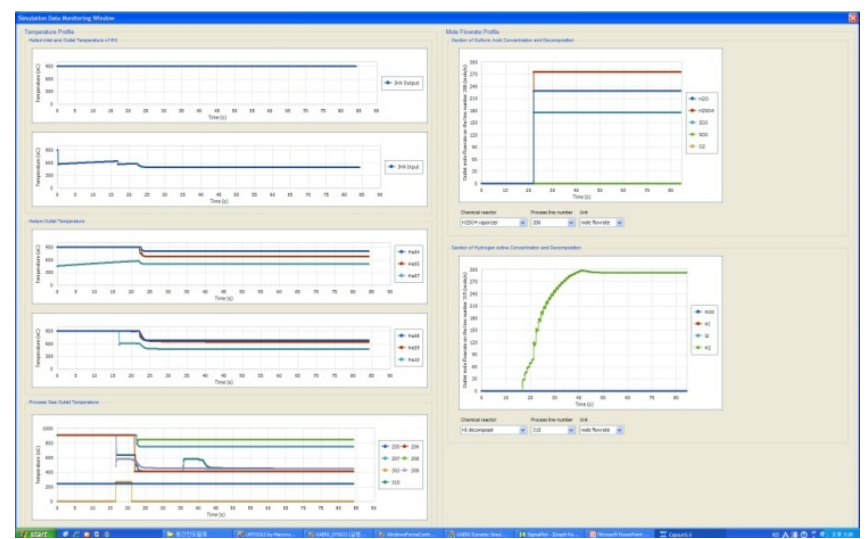

Fig. 5. Sub window of KAERI-DySCo.

As a representative example of the applied data visualization for the individual chemical reactor in KAERI-DySCo, the start-up dynamic simulation result of the HIx distillation column has been introduced.

In order to analyze the start-up dynamic behavior of the HIx distillation, the modified NRTL equation based on the Neumann model and the ideal gas law to calculate vapor-liquid equilibrium values of the $\mathrm{H}_{2} \mathrm{O}-\mathrm{HI}-\mathrm{I}_{2}$ ternary system was adopted for liquid and gas phases, respectively. The design and operation conditions for the dynamic simulation of the HIx distillation column are shown in Fig. 6. Lists of output data from the dynamic simulation are shown in Table 4 (Chang, et al., 2010).

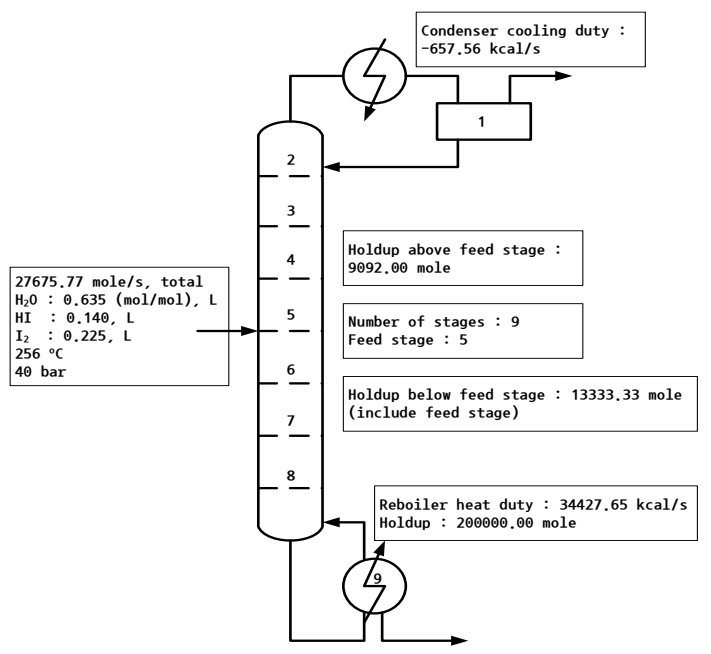

Fig. 6. Design and operation conditions for dynamic simulation of the HIx distillation process.

Table 4. Output data from the dynamic simulation of HIx distillation column

\begin{tabular}{|l|c|}
\hline \multicolumn{1}{|c|}{ Item of output data } & Unit \\
\hline $\begin{array}{l}\text { Liquid mole fraction for the } \mathrm{H}_{2} \mathrm{O}-\mathrm{HI}_{-} \mathrm{I}_{2} \text { ternary } \\
\text { system at each stage }\left(\mathrm{xH}_{2} \mathrm{O}, \mathrm{xHI}, \mathrm{xI}_{2} \text { ) }\right.\end{array}$ & - \\
\hline $\begin{array}{l}\text { Vapor mole fraction for the } \mathrm{H}_{2} \mathrm{O}-\mathrm{HI}_{-} \mathrm{I}_{2} \text { ternary } \\
\text { system at each stage }\left(\mathrm{yH}_{2} \mathrm{O}, \mathrm{yHI}, \mathrm{yI}_{2}\right)\end{array}$ & - \\
\hline Total liquid mole flow rate at each stage & mole/s \\
\hline Total vapor mole flow rate at each stage & mole/s \\
\hline Temperature at each stage & ${ }^{\circ} \mathrm{C}$ \\
\hline Boiling temperature at each stage & ${ }^{\circ} \mathrm{C}$ \\
\hline
\end{tabular}

Fig. 7 shows the results of the dynamic simulation of the HIx distillation column. In Fig. 7, the upper chart displays composition changes in each stage inside the column and the lower chart gives temperature information inside the column. The spreadsheet at the bottom of the window in Fig. 7 presents all of the listed output data in the form of numerical values.

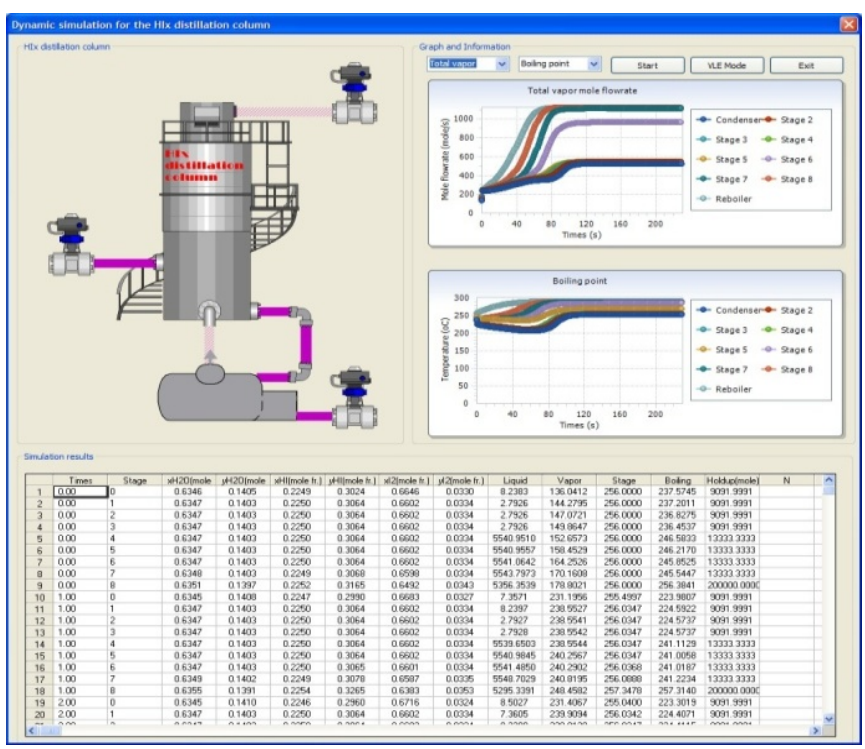

Fig. 7. Window of the dynamic simulation results of the HIx distillation column. 
KAERI-DySCo is able to display the information of vapor-liquid equilibrium curve as shown in Fig. 8.

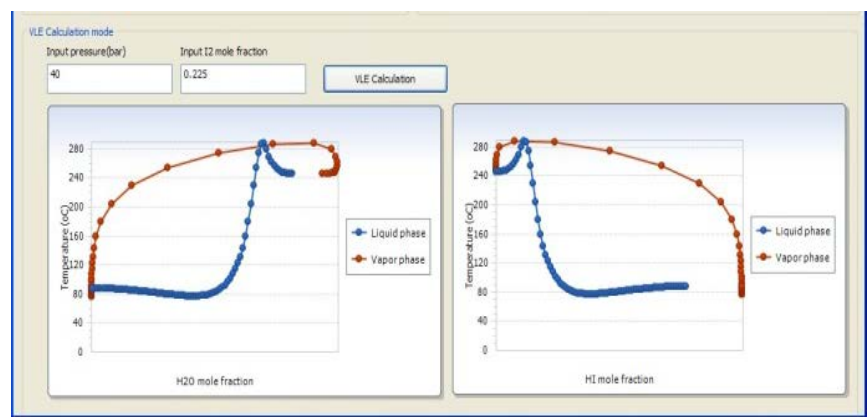

Fig. 8. Sub window for the vapor-liquid equilibrium curve of $\mathrm{H}_{2} \mathrm{O}-\mathrm{HI}-\mathrm{I}_{2}$ ternary system.

\section{Summary}

The visualization system of KAERI-DySCo for the start-up dynamic simulation of the VHTR-SI process to produce hydrogen has been developed by using the Chart FX and the Spread 7.0. The visual dynamic information displayed by a main window and sub windows of KAERI-DySCo allow users to easily analyze the start-up dynamic behavior of the SI process coupled to the VHTR.

KAERI-DySCo has been ready for validation and verification to be performed in the near future.

\section{REFERENCES}

Shin, Youngjoon, 2007, Calculation Note of the NHDD (NHDD-KA07-HP-001-00).

Kim, Jihwan, et al., 2008, International Journal of Hydrogen

Energy, Vol. 33, pp 7361-7370.

Chang, Jiwoon, et al., 2008, Korean Nuclear Society Spring Meeting, pp 109.

Microsoft Corporation, 2008, MSDN Library Visual Studio 2008 SP1 Microsoft Document Explorer.

Shin, Youngjoon, et al., 2009, Nuclear Engineering and Technology, Vol. 41, pp 831-840.

Chang, Jiwoon, et al., 2010, Korean Nuclear Society Autumn Meeting, pp 91. 